\title{
THE EFFECTS OF NONUNIFORM SURFACE TENSION \\ ON THE AXISYMMETRIC GRAVITY-DRIVEN \\ SPREADING OF A THIN LIQUID DROP
}

\author{
E. MOMONIAT
}

Received 12 October 2004

The effects of nonuniform surface tension on the axisymmetric gravity-driven spreading of a thin viscous liquid drop are investigated. A second-order nonlinear partial differential equation modelling the evolution of the free surface of a thin viscous liquid drop is derived. The nonuniform surface tension is represented by a function $\Sigma(r)$. The Lie group method is used to determine $\Sigma(r)$ such that exact and approximate invariant solutions admitted by the free surface equation can be determined. It is shown that the nonuniform surface tension can be represented as a power law in $r$. The effect of this nonuniformity is to reduce the surface tension at the centre of the drop and increase it at the foot of the drop. This results in a deflection away from the solution for spreading under gravity only and the formation of a capillary ridge.

\section{Introduction}

In this paper, the effects of nonuniform surface tension on the axisymmetric gravitydriven spreading of a thin liquid drop are investigated. Thin films have been the subject of reviews by Oron et al. [16] and Myers [12]. When the effects of surface tension are included, the free surface equation is generally a fourth-order nonlinear partial differential equation. These equations are generally very difficult to solve analytically. Analytical solutions have however been found in some cases. Pseudo-steady-state solutions are obtained in $[11,14]$. Other cases in which analytical solutions have been obtained are surveyed in Myers [12].

In this paper, we derive a second-order nonlinear partial differential equation that models the evolution of the free surface of a thin liquid drop spreading under gravity and nonuniform surface tension. Nonuniform surface tension effects have been discussed in Sherman [19]. Nonuniform surface tension effects may be caused by the presence of surfactants on the free surface of the drop. Surfactants on the free surface have a tendency to lower the free energy (surface tension) of the surface (see Figure 1.1). Surfactants on the free surface arrange themselves in such a way so as to reduce the surface tension at the centre of the axisymmetric drop and increase it at the foot of the drop. This nonuniformity in the surface tension is modelled as a function of the radial distance. $h_{o}$ is 


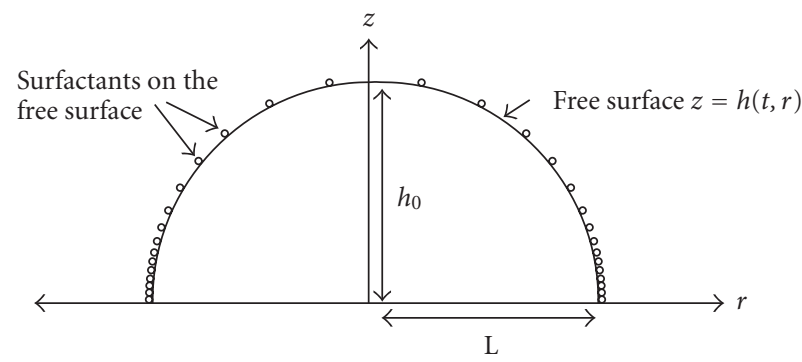

Figure 1.1. Problem configuration.

the characteristic height of the drop and $L$ the characteristic length. We model the nonuniform surface tension as a function, $\Sigma(r)$, of the radial distance. We use the Lie group method to determine functional forms of $\Sigma(r)$ such that exact and approximate groupinvariant solutions admitted by the free surface equation can be determined.

The paper is divided as follows. In Section 2, we present a brief derivation of the free surface equation. In Section 3 the Lie group method is used to determine $\Sigma(r)$ and exact solutions to the free surface equation. Approximate solutions are considered in Section 4. Concluding remarks are made in Section 5.

\section{Derivation of the governing equation}

The thin film approximations to the Navier-Stokes and continuity equations in cylindrical polar coordinates have been determined in Sherman [19] and Middleman [9]. In dimensionless form, these equations are given by

$$
\frac{\partial p}{\partial r}=\frac{\partial^{2} v_{r}}{\partial z^{2}}, \quad \frac{\partial p}{\partial z}=-1, \quad \frac{1}{r} \frac{\partial}{\partial r}\left(r v_{r}\right)+\frac{\partial v_{z}}{\partial z}=0,
$$

where $v_{r}=v_{r}(r, z, t), v_{z}=v_{z}(r, z, t)$, and $p=p(r, z, t)$ are the radial velocity, vertical velocity, and pressure terms, respectively.

In this paper, we are considering the case when the surface tension is a function of the radial distance $r$, that is, $\sigma=\sigma(r)$. We nondimensionalise the surface tension using the value of the surface tension at the foot of the drop, that is,

$$
\Sigma(r)=\frac{\sigma(r)}{\sigma(R(0))}
$$

where $R(t)$ is the radius of the drop at time $t$ and $R(0)$ is the initial drop radius. We have taken the characteristic length of the drop, $L$, to be the initial drop radius. From (2.2), this implies that

$$
\Sigma(R(0))=1
$$

We will use the initial condition (2.3) when solving for $\Sigma(r)$ using the Lie group method. 
The boundary conditions at the surface $z=0$ are given by

$$
v_{r}(r, 0, t)=0, \quad v_{z}(r, 0, t)=0
$$

Conditions (2.4) imply that there is no slip and that the fluid does not penetrate the substrate. The boundary conditions at the free surface $z=h(r, t)$ are now given by

$$
\begin{gathered}
p(r, h, t)=p_{o}-\frac{1}{B o} \Sigma(r)\left(\frac{\partial^{2} h}{\partial r^{2}}+\frac{1}{r} \frac{\partial h}{\partial r}\right), \\
\left.\frac{\partial v_{r}}{\partial z}\right|_{z=h(t, r)}=\frac{1}{\delta^{2} B o} \frac{d \Sigma(r)}{d r} \\
v_{z}(r, h, t)=\frac{\partial h}{\partial t}+v_{r}(r, h, t) \frac{\partial h}{\partial r} .
\end{gathered}
$$

$p_{o}$ is the atmospheric pressure, $B o=\rho g R(0)^{2} / \sigma(R(0))$ is the Bond number, $g$ the gravitational constant, and $\rho$ is the fluid density. $\delta=h_{o} / R(0)$, is the aspect ratio of the thin liquid drop where for thin films (see, e.g., Acheson [1]) we have that

$$
\delta^{2} \ll 1, \quad \delta^{2} \operatorname{Re} \ll 1 .
$$

$\operatorname{Re}=U R(0) / \nu$ is the Reynolds number, $U=g h_{o}^{3} /(\nu R(0))$ the characteristic horizontal velocity, and $v$ is the kinematic viscosity.

Boundary conditions (2.5) and (2.6) occur as a result of the nonuniform surface tension, $\Sigma(r)$, acting on the free surface. Condition (2.7) occurs as result of the fact that any particle on the free surface must remain on the free surface for the duration of the fluid motion. Solving (2.1) subject to (2.4)-(2.7) we obtain the free surface equation

$$
\frac{\partial h}{\partial t}=\frac{1}{3 r} \frac{\partial}{\partial r}\left(r h^{3} \frac{\partial h}{\partial r}-\alpha r h^{3} \frac{\partial}{\partial r}\left(\Sigma(r)\left(\frac{\partial^{2} h}{\partial r^{2}}+\frac{1}{r} \frac{\partial h}{\partial r}\right)\right)-\frac{3}{2} \beta r h^{2} \frac{d \Sigma(r)}{d r}\right)
$$

where

$$
\alpha=\frac{1}{B o}, \quad \beta=\frac{1}{\delta^{2} B o} .
$$

Since we have that $\alpha=O\left(\delta^{2}\right)<\beta$, we can ignore the terms in (2.9) which are coefficients of $\alpha$. Hence we are concerned with solving

$$
\frac{\partial h}{\partial t}=\frac{1}{3 r} \frac{\partial}{\partial r}\left(r h^{3} \frac{\partial h}{\partial r}-\frac{3}{2} \beta r h^{2} \frac{d \Sigma(r)}{d r}\right) .
$$

In this paper, we will concern ourselves with solving (2.11). Retaining the terms with coefficient $\alpha$ (curvature terms) from (2.9) is important when $\Sigma(r)$ is constant or small. In future work we will consider (2.9) and consider flows where $\alpha$ is of the same or greater magnitude than $\beta$. 


\section{Group-invariant solutions}

In this section, we briefly discuss how to determine Lie point symmetry generators admitted by (2.11). We use these generators to reduce (2.11) to two ordinary differential equations. One for the case $\Sigma(r)=(r / R(0))^{n}$ where $n \neq 4 / 3$ and the second for $n=4 / 3$. These ordinary differential equations are solved analytically and numerically.

We are looking for transformations of the independent variables $t, r$ and the dependent variable $h$ of the form

$$
\bar{t}=\bar{t}(t, r, h, a), \quad \bar{r}=\bar{r}(t, r, h, a), \quad \bar{h}=\bar{h}(t, r, h, a) .
$$

The transformations (3.1) form a group where $a$ is the group parameter and leave (2.11) form invariant. Lie's theory reduces the determination of the transformations (3.1) to the calculation of the infinitesimal transformations

$$
\bar{t}=t+a \xi^{1}(t, r, h), \quad \bar{r}=r+a \xi^{2}(t, r, h), \quad \bar{h}=h+a \eta(t, r, h) .
$$

The operator

$$
X=\xi^{1}(t, r, h) \partial_{t}+\xi^{2}(t, r, h) \partial_{r}+\eta(t, r, h) \partial_{h}
$$

is known as the Lie point symmetry generator admitted by (2.11). We define $\partial_{t}=\partial / \partial t, \ldots$ The functions $\xi^{1}, \xi^{2}$, and $\eta$ are calculated by solving a determining equation

$$
\left.X^{[2]}\left(\frac{\partial h}{\partial t}-\frac{1}{3 r} \frac{\partial}{\partial r}\left(r h^{3} \frac{\partial h}{\partial r}-\frac{3}{2} \beta r h^{2} \frac{d \Sigma(r)}{d r}\right)\right)\right|_{(2.11)}=0
$$

The operator $X^{[2]}$ is the second prolongation of the operator $X$ given by

$$
X^{[2]}=X+\zeta^{1} \partial_{h_{t}}+\zeta^{2} \partial_{h_{r}}+\zeta^{22} \partial_{h_{r r}},
$$

where

$$
\begin{gathered}
\zeta^{i}=D_{i} \eta-\left(D_{i} \xi^{j}\right) h_{j}, \quad i=1,2, \\
\zeta^{i_{1} i_{2} \cdots i_{k}}=D_{i_{k}} \eta_{i_{1} i_{2} \cdots i_{k-1}}-\left(D_{i_{k}} \xi^{j}\right) h_{i_{1} i_{2} \cdots j}, \quad i_{s}=2, s=1,2 .
\end{gathered}
$$

We have adopted the Einstein convention in (3.6)-(3.7). The operators, $D_{i}$, are the operators of total differentiation where

$$
\begin{aligned}
& D_{1}=D_{t}=\partial_{t}+h_{t} \partial_{h}+h_{t t} \partial_{h_{t}}+h_{t r} \partial_{h_{r}}+\cdots, \\
& D_{2}=D_{r}=\partial_{r}+h_{r} \partial_{h}+h_{t r} \partial_{h_{t}}+h_{r r} \partial_{h_{r}}+\cdots
\end{aligned}
$$

The determining equation (3.4) is separated by coefficients of derivatives of $h$ where $h_{t}$ is given by (2.11). The interested reader is referred to the books by Bluman and Kumei [5], Ibragimov [2, 7, 8], Olver [15], and Ovsiannikov [17, 18] for more information on the Lie group approach for solving differential equations. Most of the calculations described above can be performed using symbolic computer packages (see, e.g., $[4,6,20]$ ). 
When separating out by derivatives of the dependent variable and solving the resulting overdetermined system of equations, we find that

$$
X=\left(a_{2}+a_{1} t\right) \partial_{t}+a_{3} r \partial_{r}-\frac{1}{3}\left(a_{1}-2 a_{3}\right) h \partial_{h},
$$

where $a_{1}, a_{2}$, and $a_{3}$ are arbitrary constants and $\Sigma(r)$ must satisfy the ordinary differential equations

$$
\begin{gathered}
3 a_{3} r \frac{d^{2} \Sigma}{d r^{2}}-\left(a_{3}-2 a_{1}\right) \frac{d \Sigma}{d r}=0, \\
3 a_{3} r^{2} \frac{d^{3} \Sigma}{d r^{3}}+r\left(2 a_{1}+5 a_{3}\right) \frac{d^{2} \Sigma}{d r^{2}}+\left(2 a_{1}-a_{3}\right) \frac{d \Sigma}{d r}=0 .
\end{gathered}
$$

We cannot have $a_{3}=0$ since this would imply a constant solution from (3.10). Solving the system (3.10) and (3.11) for the constants $a_{1} \neq 0$ and $a_{3} \neq 0$ subject to (2.3) we find that

$$
\Sigma(r)=\left(\frac{r}{R(0)}\right)^{n}, \quad n>0
$$

when

$$
a_{1}=-\frac{1}{2} a_{3}(3 n-4)
$$

The Lie point symmetry generator (3.9) becomes

$$
X=\left(a_{2}+\frac{1}{2} a_{3}(4-3 n) t\right) \partial_{t}+a_{3} r \partial_{r}+\frac{a_{3} n}{2} h \partial_{h}
$$

The requirement that $n>0$ is imposed to ensure that there is no singular behaviour at $r=0$.

Choosing $a_{1}=0$ in (3.10) and (3.11) and solving we obtain the solution

$$
\Sigma(r)=\left(\frac{r}{R(0)}\right)^{4 / 3} .
$$

The Lie point symmetry generator (3.9) simplifies to

$$
X=a_{2} \partial_{t}+a_{3} r \partial_{r}+\frac{2}{3} a_{3} h \partial_{h}
$$

A group-invariant solution, $h=\Phi(t, r)$, admitted by (2.11) can be determined by solving the first-order quasilinear partial differential equation

$$
\left.X(h-\Phi(t, r))\right|_{h=\Phi(t, r)}=0 .
$$

Imposing (3.14) on (3.17) we find that

$$
\begin{gathered}
h(t, r)=\left(2 a_{2}+a_{3}(4-3 n) t\right)^{n /(4-3 n)} H(p), \\
p=r\left(2 a_{2}+a_{3}(4-3 n) t\right)^{-2 /(4-3 n)},
\end{gathered}
$$


708 Nonuniform surface tension effects

where

$$
n \neq \frac{4}{3}
$$

Substituting (3.18) with (3.12) into (2.11) we reduce (2.11) to the second-order ordinary differential equation

$$
\begin{aligned}
\frac{d^{2} H}{d p^{2}}=\left(2 p^{2} H^{3}\right)^{-1}[ & 3 \beta n\left(\frac{p}{R(0)}\right)^{n} H\left(n H+2 p \frac{d H}{d p}\right) \\
& \left.-2 p\left(-3 a_{3} n p H+\left(6 a_{3} p^{2}+H^{3}\right) \frac{d H}{d p}+3 p H^{2}\left(\frac{d H}{d p}\right)^{2}\right)\right] .
\end{aligned}
$$

The ordinary differential equation (3.20) admits the Lie point symmetry generator

$$
Y=\frac{2}{n} p \partial_{p}+H \partial_{H}
$$

Therefore, we find that the ordinary differential equation (3.20) admits the group-invariant solution

$$
H(p)=\sqrt{3 \beta}\left(\frac{p}{R(0)}\right)^{n / 2} .
$$

Therefore, from (3.18) we find that

$$
h(t, r)=\sqrt{3 \beta}\left(\frac{r}{R(0)}\right)^{n / 2}
$$

which is a nonphysical stationary solution.

We now proceed to solving (3.20) numerically. Imposing the boundary condition

$$
h(t, R(t))=0
$$

on (3.18) we find that

$$
R(t)=\kappa\left(2 a_{2}+a_{3}(4-3 n) t\right)^{2 /(4-3 n)}
$$

where $\kappa$ is a constant. Therefore, we have to solve (3.20) subject to the boundary condition

$$
H(\kappa)=0
$$




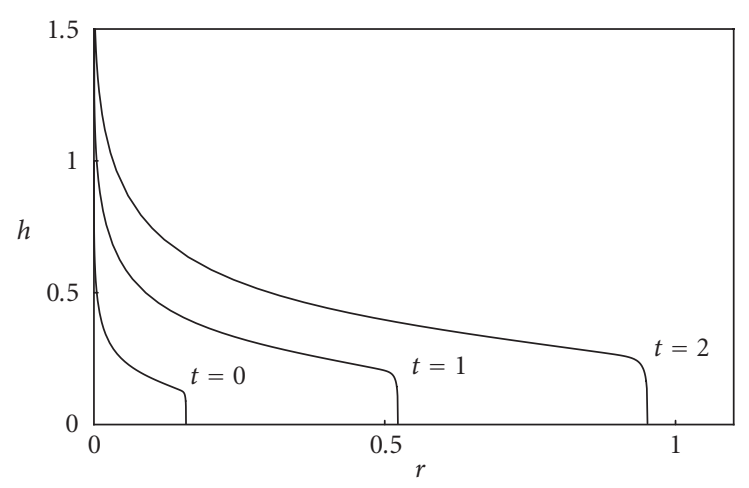

Figure 3.1. Plot of nonphysical solution satisfying only the condition that the radius be increasing. This contradicts the requirement that the drop height be decreasing. The plot shows an increasing drop height for increasing radius.

Also, we normalise the initial drop height such that

$$
h(0,0)=1 .
$$

Therefore

$$
H(0)=\left(2 a_{2}\right)^{-n /(4-3 n)} .
$$

We have two boundary conditions so we do not need to use (3.21) to effect a further reduction of (3.20). To obtain physically meaningful solutions we need the coefficient of $H$ in (3.18) to have a negative exponent so that the drop height is decreasing. Therefore we must have $n /(4-3 n)<0 \rightarrow n>4 / 3$. But we also need $R(t)$ to be increasing so that the drop is spreading. This is not possible, since if $n>4 / 3$, then $R(t)$ is a decreasing function. The only alternative is a stationary drop. This would imply that $n=4 / 3$ or $a_{3}=0$. The case $n=4 / 3$ is not valid as we would get division by 0 . Therefore we cannot obtain a physically meaningful solution by solving (3.20) numerically using the boundary conditions (3.26) and (3.28). The group-invariant solution (3.18) is plotted in Figure 3.1 where (3.20) has been solved numerically subject to (3.26) and (3.28). Here we have chosen $a_{2}=1 / 2, a_{3}=$ $1, n=0.8, \beta=1$, and $\kappa=0.1582975$. We observe from Figure 3.1 that this solution is not physical.

We now plot the stationary solution by choosing $a_{3}=0$ and $a_{2}=1 / 2$. The numerical algorithm from Mathematica can only satisfy $H\left(10^{-10}\right)=0.988338$ where $n=0.8, \beta=1$, and $\kappa=11.5$. We are unable to get a better result. The graph is shown in Figure 3.2.

The group-invariant solution corresponding to the Lie point symmetry generator (3.16) is given by

$$
h(t, r)=\exp \left(\frac{2 a_{3}}{3 a_{2}} t\right) G(q), \quad q=r \exp \left(-\frac{a_{3}}{a_{2}} t\right) .
$$


710 Nonuniform surface tension effects

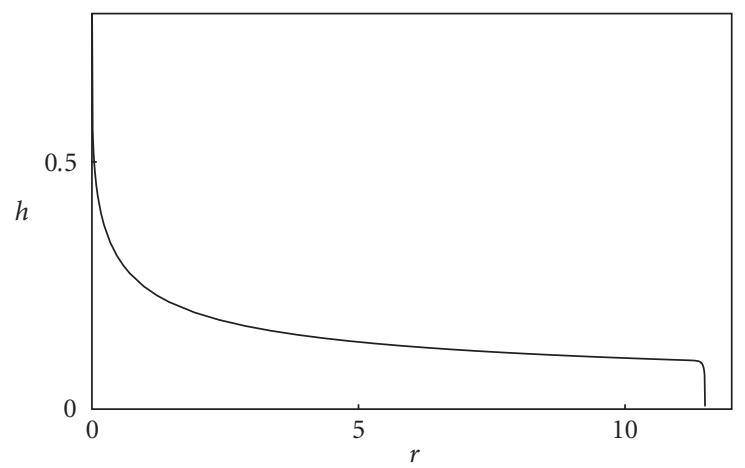

Figure 3.2. Plot of stationary solution for $n=0.8$ satisfying the boundary conditions $H(11.5)=10^{-3}$ and $H\left(10^{-10}\right)=0.988338$.

Substituting (3.29) with (3.15) into (2.11) we obtain the second-order ordinary differential equation

$$
\begin{aligned}
\frac{d^{2} G}{d q^{2}}=\left(3 a_{2} q R(0) G^{3}\right)^{-1} & {\left[6 a_{3} q R(0) G-3 R(0)\left(3 a_{3} q^{2}+a_{2} G^{3}\right) \frac{d G}{d q}-9 a_{2} q R(0) G^{2}\left(\frac{d G}{d q}\right)^{2}\right.} \\
& \left.+4 a_{2} \beta\left(\frac{q}{R(0)}\right)^{1 / 3} G\left(2 G+3 q \frac{d G}{d q}\right)\right]
\end{aligned}
$$

The ordinary differential equation (3.30) admits the Lie point symmetry generator

$$
Z=\frac{3}{2} p \partial_{p}+H \partial_{H}
$$

Hence the ordinary differential equation (3.30) admits a group-invariant solution

$$
G(q)=\sqrt{3 \beta}\left(\frac{q}{R(0)}\right)^{2 / 3} .
$$

Therefore, from (3.29) we find that

$$
h(t, r)=\sqrt{3 \beta}\left(\frac{r}{R(0)}\right)^{2 / 3}
$$

which is a nonphysical stationary solution. 


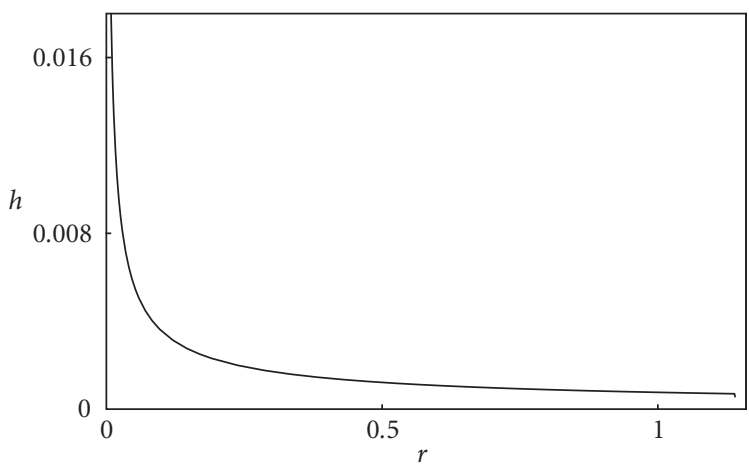

Figure 3.3. Plot of stationary solution for $n=3 / 4$ satisfying the boundary conditions $G(1.14)=10^{-4}$ and $G\left(10^{-4}\right)=0.0865109$.

We now try to solve (3.30) numerically. Imposing the boundary condition (3.24) and (3.27) on (3.29) we find that

$$
\begin{gathered}
R(t)=\lambda \exp \left(\frac{a_{3}}{a_{2}} t\right), \\
G(\lambda)=0, \quad G(0)=1,
\end{gathered}
$$

where $\lambda$ is an arbitrary constant. Again, we cannot obtain a physically meaningful solution by solving (3.30) numerically because of the incompatibility of the boundary conditions. We need to satisfy decreasing drop height, that is, $2 a_{3} /\left(3 a_{2}\right)<0$. But this implies that the radius is decreasing from (3.34). We plot a stationary solution in Figure 3.3 by choosing $a_{3}=0, a_{2}=1, \beta=1$, and $\lambda=1.14$. Numerically, we find that we can only impose the boundary conditions $G(\lambda)=10^{-4}$ and $G\left(10^{-4}\right)=0.0865109$.

\section{Approximate solutions for thin film flow}

In this section, we make the assumption that $\beta$ is small. We determine approximate solutions to (2.11) of the form

$$
h(t, r)=h_{0}(t, r)+\beta h_{1}(t, r)+\cdots,
$$

where

$$
\beta \ll 1
$$

Substituting (4.1) into (2.11) and separating (2.11) by coefficients to $O(1)$ and $O(\beta)$, we obtain the system of partial differential equations

$$
\begin{gathered}
\frac{\partial h_{0}}{\partial t}=\frac{1}{3 r} \frac{\partial}{\partial r}\left(r h_{0}^{3} \frac{\partial h_{0}}{\partial r}\right) \\
\frac{\partial h_{1}}{\partial t}=\frac{1}{3 r} \frac{\partial}{\partial r}\left(r \frac{\partial}{\partial r}\left(h_{0}^{3} h_{1}\right)-\frac{3}{2} r h_{0}^{2} \frac{d \Sigma}{d r}\right) .
\end{gathered}
$$


712 Nonuniform surface tension effects

The solution admitted by (4.3) is given by

$$
h_{0}(t, r)=\frac{1}{R^{2}(t)}\left(1-\frac{r^{2}}{R^{2}(t)}\right)^{1 / 3},
$$

where

$$
R(t)=\left(1+\frac{16}{9} t\right)^{1 / 8}
$$

has been determined by Barenblatt [3]. Equation (4.6) implies that (3.12) becomes

$$
\Sigma(r)=r^{n}
$$

Recently, Momoniat et al. [10] have successfully used the Lie group method to obtain (4.5) and (4.6). Substituting (4.5) with (4.6) into (4.4) and using the Lie symmetry approach to analyse the resulting equation we find that the resulting equation admits the Lie point symmetry

$$
Y=\left(1+\frac{16}{9} t\right) \partial_{t}+\frac{2}{9} r \partial_{r}+\frac{2}{9}(n+2) h_{1} \partial_{h_{1}}
$$

where (3.12) holds. The group-invariant solution corresponding to (4.8) is given by

$$
h_{1}(t, r)=R^{n+2}(t) H_{1}(q), \quad q=\frac{r}{R(t)} .
$$

This solution is valid for $n=4 / 3$. Substituting (4.9) into (4.4) we obtain the second-order ordinary differential equation

$$
\begin{aligned}
\frac{d^{2} H_{1}}{d p^{2}}=-\left(6 p^{2}\left(1-p^{2}\right)^{4 / 3}\right)^{-1} & {\left[3 n p^{n}\left(-3 n+(4+3 n) p^{2}\right)\right.} \\
& \left.-2 p\left(1-p^{2}\right)^{1 / 3}\left(2(8+n) p H_{1}+\left(13 p^{2}-3\right) \frac{d H_{1}}{d p}\right)\right] .
\end{aligned}
$$

Imposing the boundary conditions

$$
h(t, R(t))=0, \quad h(0,0)=1,
$$

we find that (4.10) is solved numerically subject to

$$
H_{1}(0)=0, \quad H_{1}(1)=0 .
$$

However, the ordinary differential equation (4.10) has singular behaviour at $p=0$ and $p=1$. We impose the conditions (4.12) at $p=10^{-6}$ and 0.999999 . Therefore, we have that

$$
H_{1}\left(10^{-6}\right)=0, \quad H_{1}(0.999999)=0 .
$$




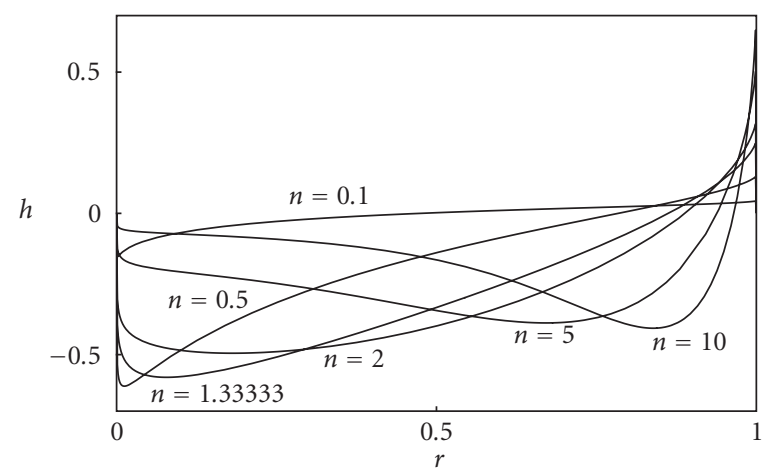

Figure 4.1. Numerical solution of the ordinary differential equation for the perturbed solution for different values of $n$ including $n=4 / 3$.

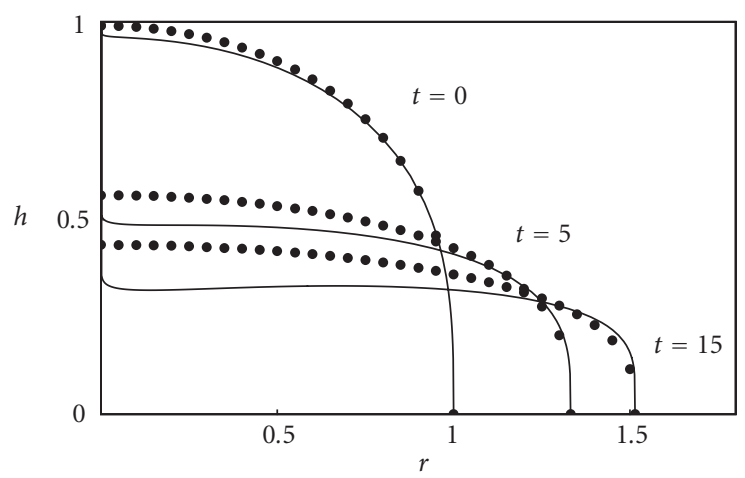

Figure 4.2. Plot comparing the solution for spreading under gravity only $(\bullet \bullet \bullet)$ with the solution for spreading under gravity and nonuniform surface tension where $\Sigma(r)=r^{4 / 3}$ taking $\beta=0.05$.

We use a shooting method to solve (4.10) subject to (4.13). The numerical solution obtained from Mathematica is shown in Figure 4.1 for different values of $n$. We observe from Figure 4.1 that as $n$ increases the capillary ridge becomes more pronounced. Also, as $n$ increases the position of the deflection moves away from the origin. This can be seen in Figures 4.2 and 4.3 where we have plotted the solution for spreading under gravity only (4.5) and the approximate solution (4.1) on the same system of axis for different values of $n$. In Figure 4.2, a deflection of the approximate solution away from the solution for spreading under gravity only is indicated. The defelection occurs close to the origin. The formation of a capillary ridge cannot be seen. In Figure 4.3, both the deflection and capillary ridge can be clearly seen. The deflection occurs further away from the origin.

\section{Concluding remarks}

In this paper, we have used the Lie group method to determine a power law expression for the nonuniform surface tension, that is, $\Sigma(r)=(r / R(0))^{n}$. We have shown that the exact 


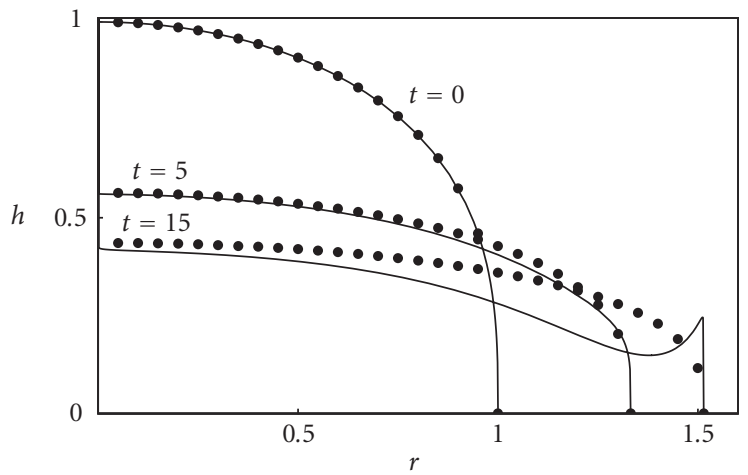

Figure 4.3. Plot comparing the solution for spreading under gravity only $(\bullet \bullet \bullet)$ with the solution for spreading under gravity and nonuniform surface tension where $\Sigma(r)=r^{10}$ taking $\beta=0.002$.

Lie group approach yields only nonphysical or stationary solutions. The approximate Lie group approach yields a physically meaningful solution showing a deflection away from the solution for spreading under gravity only. We also observe the formation of a capillary ridge at the leading edge of the liquid drop. This is a well-known effect of surface tension (see, e.g., Myers and Charpin [13]). Further research is required to relate the exponent in the power law representation of $\Sigma(r)$ to some physically relevant surfactants.

\section{Acknowledgments}

I would like to thank T. G. Myers, S. Abelman, and D. P. Mason for helpful comments. This material is based upon work supported by the National Research Foundation, South Africa, under Grant 2053745.

\section{References}

[1] D. J. Acheson, Elementary Fluid Dynamics, Oxford Applied Mathematics and Computing Science Series, The Clarendon Press, Oxford University Press, New York, 1990.

[2] W. F. Ames, R. L. Anderson, V. A. Dorodnitsyn, E. V. Ferapontov, R. K. Gazizov, N. H. Ibragimov, and S. R. Svirshchevskiü, CRC Handbook of Lie Group Analysis of Differential Equations. Vol. 1, edited by N. H. Ibragimov, CRC Press, Florida, 1994.

[3] G. I. Barenblatt, On certain non-steady motions of fluids and gases in porous media, Prikl. Mat. Mekh. 16 (1952), no. 1, 67-78.

[4] G. Baumann, Symmetry Analysis of Differential Equations with Mathematica, Springer, New York, 2000.

[5] G. W. Bluman and S. Kumei, Symmetries and Differential Equations, Applied Mathematical Sciences, vol. 81, Springer, New York, 1989.

[6] A. K. Head, LIE, a PC program for Lie analysis of differential equations, Comput. Phys. Comm. 77 (1993), no. 2, 241-248.

[7] N. H. Ibragimov, Elementary Lie Group Analysis and Ordinary Differential Equations, Wiley Series in Mathematical Methods in Practice, vol. 4, John Wiley \& Sons, Chichester, 1999.

[8] N. H. Ibragimov, A. V. Aksenov, V. A. Baikov, V. A. Chugunov, R. K. Gazizov, and A. G. Meshkov, CRC Handbook of Lie Group Analysis of Differential Equations. Vol. 2, edited by N. H. Ibragimov, CRC Press, Florida, 1995. 
[9] S. Middleman, Modelling Axisymmetric Flows: Dynamics of Films, Jets, and Drops, Academic Press, New York, 1995.

[10] E. Momoniat, D. P. Mason, and F. M. Mahomed, Non-linear diffusion of an axisymmetric thin liquid drop: group-invariant solution and conservation law, Internat. J. Non-Linear Mech. 36 (2001), no. 6, 879-885.

[11] J. A. Moriarty, L. W. Schwartz, and E. O. Tuck, Unsteady spreading of thin liquid films with small surface tension, Phys. Fluids A 3 (1991), no. 5, 733-742.

[12] T. G. Myers, Thin films with high surface tension, SIAM Rev. 40 (1998), no. 3, 441-462.

[13] T. G. Myers and J. P. F. Charpin, The effect of the Coriolis force on axisymmetric rotating thin film flows, Internat. J. Non-Linear Mech. 36 (2000), no. 4, 629-635.

[14] T. G. Myers, J. P. F. Charpin, and C. P. Thompson, Slowly accreting ice due to supercooled water impacting on a cold surface, Phys. Fluids 14 (2002), no. 1, 240-256.

[15] P. J. Olver, Applications of Lie Groups to Differential Equations, Graduate Texts in Mathematics, vol. 107, Springer, New York, 1986.

[16] A. Oron, S. H. Davis, and S. G. Bankoff, Long-scale evolution of thin liquid films, Rev. Modern Phys. 69 (1997), no. 3, 931-980.

[17] L. V. Ovsiannikov, Group Properties of Differential Equations, Izdat. Sibirsk. Otdel. Akad. Nauk SSSR, Novosibirsk, 1962.

[18]_ Group Analysis of Differential Equations, Academic Press, New York, 1982.

[19] F. S. Sherman, Viscous Flow, McGraw-Hill, New York, 1990.

[20] J. Sherring, A. K. Head, and G. E. Prince, Dimsym and LIE: symmetry determination packages, Math. Comput. Modelling 25 (1997), no. 8-9, 153-164.

E. Momoniat: Centre for Differential Equations, Continuum Mechanics and Applications, School of Computational and Applied Mathematics, University of the Witwatersrand, Private Bag 3, Wits 2050, Johannesburg, South Africa

E-mail address: ebrahim@cam.wits.ac.za 


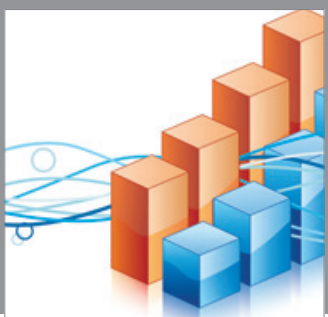

Advances in

Operations Research

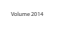

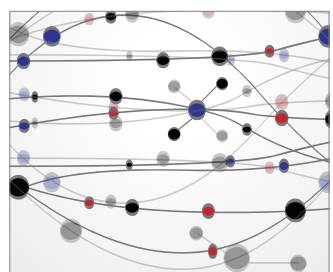

\section{The Scientific} World Journal
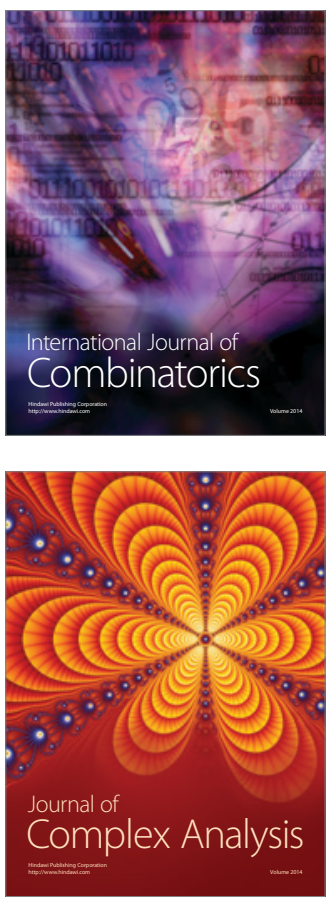

International Journal of

Mathematics and

Mathematical

Sciences
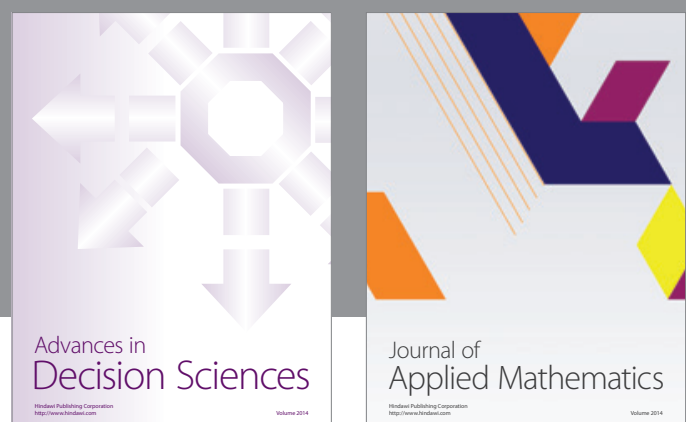

Journal of

Applied Mathematics
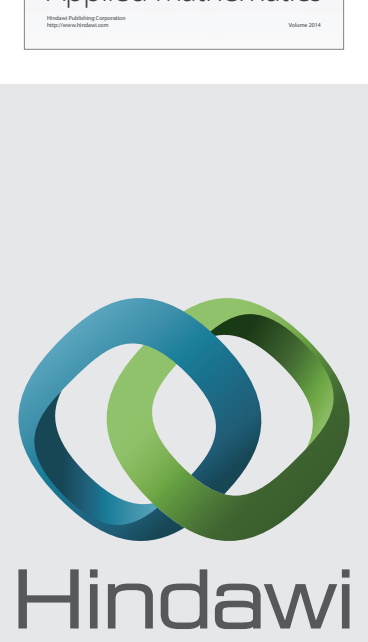

Submit your manuscripts at http://www.hindawi.com
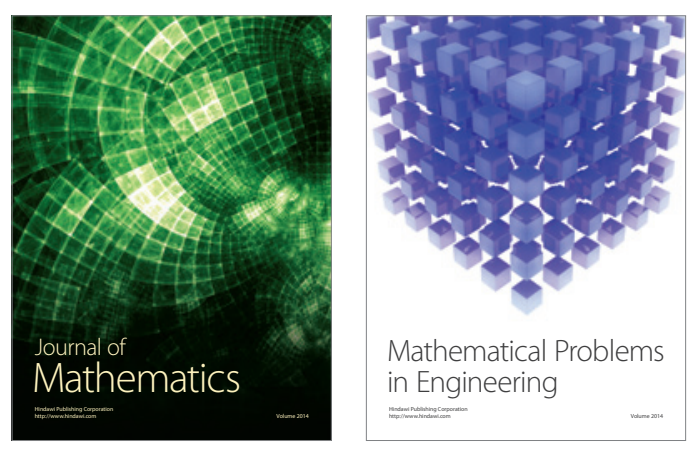

Mathematical Problems in Engineering
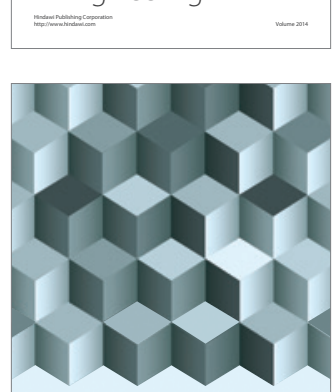

Journal of

Function Spaces
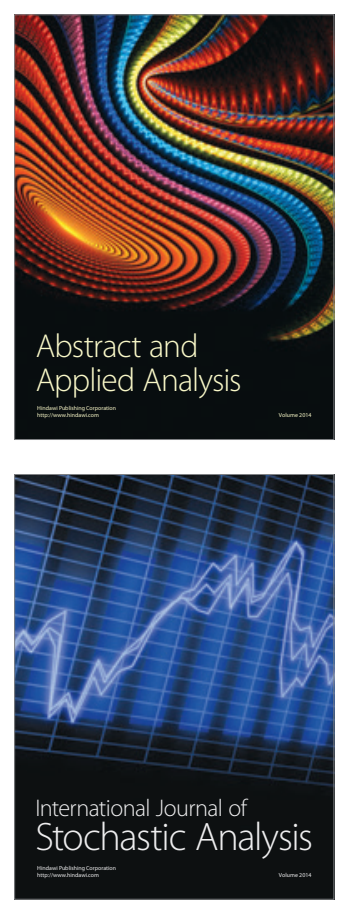

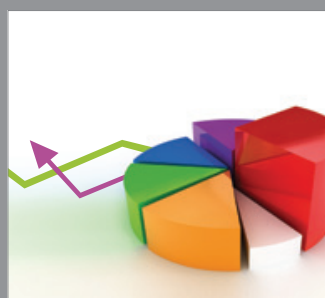

ournal of

Probability and Statistics

Promensencen
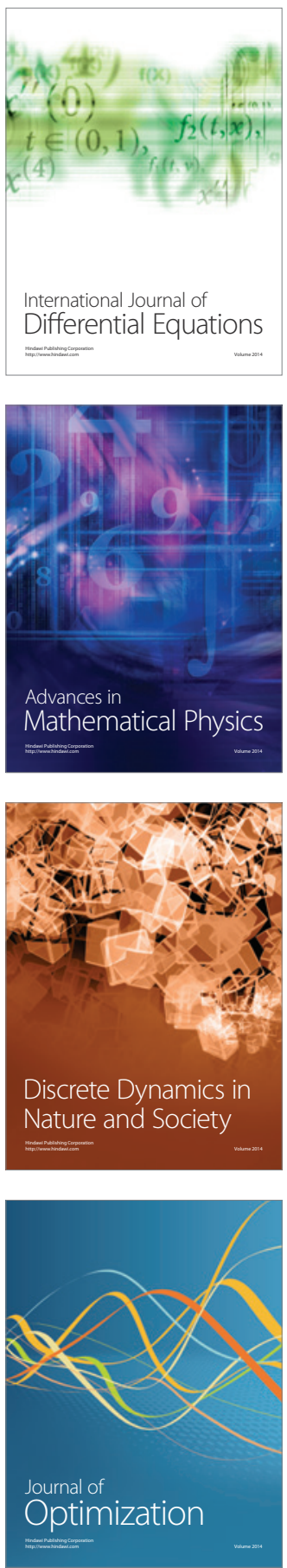